\title{
¿Democratiza el sorteo la democracia? Cómo la democracia deliberativa ha despolitizado una propuesta radical*
}

\author{
Does random selection make democracies more democratic? \\ How deliberative democracy has depoliticized a radical proposal
}

JULIEN TALPIN ${ }^{* *}$

\begin{abstract}
Resumen: El regreso del sorteo a la política desde hace cuarenta años debe mucho a su apropiación por parte de las teorías de la democracia deliberativa, que han hecho de los dispositivos sorteados los espacios centrales de la deliberación democrática. Esta apropiación, sin embargo, no era en absoluto evidente. Tiene que ver con la trayectoria científica de algunos de sus promotores y con evoluciones paralelas en el seno del campo político. A pesar del aire fresco que ha insuflado al gobierno representativo, la promoción científica del sorteo no se ha traducido necesariamente en una democratización de la democracia. Estos experimentos contribuyen a demostrar las capacidades deliberativas de los ciudadanos ordinarios, pero tan solo en casos excepcionales han contribuido a incrementar su poder en la toma de decisiones. La focalización de las investigaciones sobre el análisis de las dinámicas deliberativas en el interior de dispositivos sorteados ha sido, por tanto, muy criticado por estudiosos que reivindican un retorno a la inspiración inicial habermasiana de una deliberación más amplia en el espacio público, no restringida al interior de los mini-públicos. Este giro sistémico ha marginado el sorteo en la teoría deliberativa
\end{abstract}

\begin{abstract}
Random selection grand come-back in politics in the last forty years is partly due to its incorporation by the theories of deliberative democracy, that have made of randomly selected devices the central forums of deliberation. This integration was, however, far from being selfevident. It stemmed from the scientific trajectory of its proponents and from parallel trends in the political field. Despite, the fresh air random selection brings to representative government, its scientific promotion has not necessarily meant a democratization of democracy however. While such experiments have demonstrated the deliberative capacities of ordinary citizens, they have only exceptionally increased their power in decision-making processes. The focus put by research on the analysis of deliberative dynamics within randomly selected devices has therefore been harshly criticized by some deliberativists who reclaim a needed come back to Habermas' initial inspiration of a greater deliberation in the public sphere rather than confined in mini-publics. This systemic turn has marginalized random selection within the most recent deliberative theories. After tracing back
\end{abstract}

Fecha de recepción: 01/06/2017. Fecha de aceptación: 19/09/2017.

* La traducción al español de este texto, a cargo de Jorge Costa Delgado, se ha hecho en el marco del proyecto de I+D "La recepción de la filosofía grecorromana en la filosofía y las ciencias humanas en Francia y España desde 1980 hasta la actualidad" (FFI2014-53792-R).

** Investigador en el Centre National de la Recherche Scientifique (CNRS) en la Universidad de Lille (Francia). Líneas de investigación: Sociología política, Teoría política, Sociología urbana. Publicaciones recientes: Community Organizing. De l'émeute à l'alliance des classes populaires à Los Angeles, París, Raisons d'agir, 2016; "What can ethnography bring to the study of deliberative democracy? Evidence from the study on the impact of participation on actors", Revista Internacional de Sociología, 70 (2), 2012, p. 143-163.

Contacto: julien.talpin@univ-lille2.fr 
más reciente. Después de reconstruir este recorrido intelectual, me gustaría esbozar algunos elementos para reflexionar sobre la manera en que el sorteo podría contribuir a reanimar la llama crítica de la teoría deliberativa, mediante su uso como herramienta de oposición por parte de los movimientos sociales.

Palabras clave: Sorteo, Democracia deliberativa, Teoría crítica, Participación política, Post-democracia. this intellectual path, I sketch some arguments about the way random selection could rekindle the critical spirit of deliberative theory, mainly through its oppositional use by social movement. Keywords: Sortition, Deliberative democracy, Critical theory, Political participation, Postdemocracy.

El sorteo encarna un principio radical de igualdad absoluta de todos los ciudadanos. El recurso al sorteo en política supone considerar, si no que la competencia política es universal, al menos que cada individuo dispone de suficiente sentido común y de interés general para decidir sobre cuestiones importantes (Finley, 1976; Rancière, 2005; Sintomer, 2007). El interés contemporáneo por este mecanismo democrático se debe en parte a esta radicalidad: viene a romper con la naturalización de la delegación política, a desestabilizar una concepción de la democracia fundada en la desigualdad en cuanto a las capacidades de cada individuo y a recordar el principio cardinal de la igualdad.

El sorteo ha protagonizado, desde hace cuarenta años, un regreso inesperado a la política, debido a razones sociales, técnicas y políticas específicas (Stone, 2009; Sintomer, 2012). Esta renovación se debe, en parte, a su apropiación por parte de las teorías de la democracia deliberativa, que a partir de los años noventa hicieron de los dispositivos sorteados los espacios centrales de la deliberación democrática. La figura de James Fishkin, a la vez teórico político y creador de un dispositivo deliberativo sorteado que tendrá gran repercusión, la encuesta deliberativa, es aquí central. No solamente su empresa académica -en todos los sentidos del término- va a conocer un desarrollo importante, sino que el éxito de la encuesta deliberativa va a hacer de este dispositivo muy particular el nuevo "patrón oro" -por recuperar la expresión de Jane Mansbridge (2010) - a partir del cual se analizará la deliberación y se promoverá el sorteo. Como se verá más adelante, esta apropiación del sorteo por los partidarios de la deliberación pública no era en absoluto evidente. Se debe a la trayectoria científica de algunos de sus promotores y a evoluciones paralelas en el seno del campo político, pero no por ello dejó de tener importantes consecuencias sobre los usos contemporáneos del sorteo.

A pesar del aire fresco que ha insuflado sobre un gobierno representativo en crisis, la promoción científica del sorteo por los partidarios de una concepción deliberativa de la democracia no se ha traducido necesariamente en una democratización de la democracia (Pateman, 2012). En efecto, los usos de dispositivos sorteados asociados a la democracia deliberativa han demostrado ser, muy a menudo, bastante limitados: han permitido probar las capacidades deliberativas de los ciudadanos ordinarios, pero sin contribuir necesariamente a incrementar su poder en la toma de decisiones. La focalización de las investigaciones en el análisis de las dinámicas deliberativas en el interior de dispositivos sorteados ha sido, por tanto, muy criticada por estudiosos que reivindican un retorno a la inspiración inicial habermasiana de una deliberación más amplia en el espacio público, 
no restringida al interior de foros microscópicos (véase, en particular, a Chambers, 2009). Estas críticas se han extendido rápidamente entre los teóricos de la deliberación, que desde entonces han realizado un giro "sistémico", interesándose en las condiciones de la deliberación en diferentes espacios sociales (Parkinson y Mansbridge, 2012). Este viraje teórico ha significado la marginación reciente del sorteo en la democracia deliberativa. Después de reconstruir este recorrido intelectual, me gustaría esbozar algunos elementos para reflexionar sobre la manera en que el sorteo podría contribuir a reanimar la llama crítica de la teoría deliberativa, que se ha atenuado un poco estos últimos años.

\section{La apropiación del sorteo por la democracia deliberativa: un encuentro contingente}

El recurso al sorteo parece hoy parte integrante de la caja de herramientas con la que opera la democracia deliberativa, que está muy asociada a la organización de dispositivos llamados "mini-públicos" donde los ciudadanos sorteados deliberan durante un período determinado sobre un tema de interés público. Sin embargo, la centralidad del sorteo en la teoría deliberativa no tenía nada de evidente. Está ligada a un conjunto de contingencias teóricas y prácticas, a través de las cuales el sorteo llegó a responder a algunos de los problemas que se plantearon los teóricos de la deliberación a partir de los años ochenta.

El sorteo inicialmente no formaba parte del arsenal teórico de la democracia deliberativa. Los primeros pensadores de esta, Jürgen Habermas (1981), Bernard Manin (1985), Jon Elster (1986) o Joshua Cohen (1989), no lo mencionan directamente. Sus referencias históricas se sitúan más bien en los salones y cafés de los siglos XVII y XVIII, en las deliberaciones de las asambleas constituyentes de las revoluciones americanas y francesas o en los town meetings de Nueva Inglaterra (Cossart, Felicetti, Kloppenberg, 2016). Aunque Jon Elster (1986) hace referencia directamente a la ciudad ateniense -mediante la figura del "fórum"-, evoca la asamblea (Ecclésia), un espacio de deliberación abierto a todos los hombres, y no las instituciones sorteadas que existían en esa época. Desde su punto de vista -compartido por los demás fundadores de la teoría deliberativa-, lo que importaba era más bien la discusión y el intercambio de argumentos en el espacio público, antes que la participación de ciudadanos ordinarios en los procesos de toma de decisiones mediante el recurso al sorteo. Esta omisión es aún más sorprendente en el caso de Elster, ya que es uno de los teóricos que ha contribuido más vivamente a la recuperación del interés de las ciencias sociales por el sorteo (véase, por ejemplo, a Goodwin, 2011). En su obra de 1989 Solomonic Judgements dedica un amplio espacio a describir los procedimientos del sorteo en Atenas, Florencia y Venecia. Sin embargo, lo que le interesa de la selección aleatoria de los representantes es sobre todo la superación de ciertos límites de la racionalidad individual y colectiva. Su defensa del sorteo -en política y más allá- trata sobre todo de preguntarse por las condiciones de producción de "buenas decisiones" cuando la razón no permite alcanzarlas, en el marco de un debate con los partidarios de la elección racional. Así, el sorteo permitiría, frecuentemente, llegar a decisiones "pareto-óptimas" y evitaría la formación de minorías permanentes. Estas reflexiones sobre la formación de preferencias individuales y los límites de su agregación mediante el voto (en términos de promoción del bien común) jugarían un rol importante en el desarrollo subsiguiente de la teoría deliberativa (Elster, 1998), pero surgen al margen de una conceptualización 
propiamente democrática del lugar del sorteo en la deliberación. No es que tales reflexiones no existieran en esta época, pero eran relativamente marginales. Benjamin Barber, por ejemplo, en su obra Strong Democracy (1984, 290-293), evoca el recurso al sorteo en Atenas; pero este no constituye para él más que una reforma institucional entre otras que permiten profundizar en la democracia. Principalmente, su proyecto teórico se sitúa al margen de la conceptualización deliberativa de la democracia que comienza entonces a plantearse, asemejándose más bien a una perspectiva participativa.

Si bien las reflexiones sobre el lugar del sorteo en política se hacen más frecuentes a finales de los años ochenta, la unión entre democracia deliberativa y sorteo corresponde inicialmente a un profesor de teoría política que va a jugar aquí un papel decisivo: James Fishkin. En su obra de 1991, Democracy and Deliberation, intenta conciliar los imperativos de igualdad política entre los ciudadanos y de centralidad de la deliberación en la construcción de elecciones colectivas legítimas. Pese a que la deliberación y la participación pueden parecer contradictorias -la calidad de la deliberación puede suponer limitar la participación y, simétricamente, una participación importante complica la deliberación-, Fishkin ve en el sorteo un medio para conciliar estos bienes en tensión.

La referencia al sorteo está directamente importada de la experiencia ateniense ${ }^{1}$, siendo el proyecto de Fishkin crear una "democracia directa deliberativa" en las condiciones de los estados-nación de finales del siglo XX. Este libro "se inscribe en una búsqueda de 2500 años que pretende adaptar mejor la idea democrática, inicialmente concebida para una ciudad-estado griega de algunos miles de miembros, a los mega-estados modernos compuestos de millones de ciudadanos. Se interesa por la manera en que se pueden conciliar las virtudes de la democracia cara-a-cara y en grupos pequeños con los estados-nación de gran tamaño" (Fishkin, 1991, 1). Si bien se refiere más a los padres fundadores de la República americana o a los trabajos de Robert Dahl (1989), antes que a los de Jürgen Habermas, su ambición teórica consiste en conciliar las virtudes de la deliberación y el imperativo democrático de igualdad entre los ciudadanos. Aunque no es cierto que su trayectoria intelectual haya tomado realmente esta dirección, como veremos más adelante, Fishkin juega un rol central en la importación de la referencia del sorteo para el arsenal teórico y práctico de la democracia deliberativa. Esto va a orientar, de ahí en adelante y de manera decisiva, los debates en este campo de investigación. En particular, a partir de su obra de 1991, Fishkin propone la creación de un dispositivo deliberativo sorteado original: la encuesta deliberativa.

Esta importación se inscribe en un movimiento más largo, desde los años setenta, de recuperación del sorteo en la política, encarnado en la multiplicación de experimentaciones deliberativas como las células de planificación en Alemania, los jurados ciudadanos en los Estados Unidos y las conferencias de consenso en Dinamarca (Sintomer, 2007) . Si bien hacían falta importadores -Fishkin juega este papel, evocando algunas de estas

1 A pesar de que Fishkin comete errores de interpretación y anacronismos, cf. Girard, 2012.

2 Esta cita, como las siguientes, se traduce a partir de una traducción previa del autor del artículo al francés.

3 Los creadores de estas innovaciones democráticas, como Peter Dienel o Ned Crosby, situaban sus proyectos en una perspectiva política y tecnocrática -permitir la participación de ciudadanos en elecciones científicas y técnicas complejas- sin hacer referencia directa a la concepción deliberativa de la democracia (que no existía como tal cuando pusieron en marcha sus experimentos). 
experiencias en Democracy and Deliberation-, el giro procedimental que encarna la teoría de la democracia deliberativa constituye igualmente un terreno propicio para este encuentro con el sorteo. En efecto, la teoría deliberativa acentúa el prisma procedimental del enfoque habermasiano (Cohen, 1989). Las condiciones de una buena deliberación, que permiten que sea verdaderamente informada, inclusiva e igualitaria, están en el centro de atención. Esta inflexión aspira a responder a la crítica dirigida a Habermas según la cual, en el mundo real, la situación social de los interlocutores y las relaciones de poder en las cuales se encuentran influyen sobre la deliberación, por lo que esta difícilmente podría reducirse a la fuerza del mejor argumento (Sintomer, 2011). Los procedimientos y la manera de regular las interacciones discursivas apuntan, de esta manera, a neutralizar las distorsiones producidas por las desigualdades sociales.

Los mini-públicos - por retomar la expresión forjada por Archon Fung (2003) para caracterizar estos diferentes dispositivos sorteados- parecen poder encarnar este giro procedimental, controlando el peso de las desigualdades sociales y culturales sobre la comunicación democrática. Su éxito, tanto académico como político, no puede sin embargo comprenderse únicamente a la luz de estas consideraciones teóricas. Estos dispositivos experimentales son igualmente coherentes con los llamamientos cada vez más apremiantes, a partir de los años noventa, a probar empíricamente las teorías deliberativas, ya sea estudiando la dinámica de las discusiones o los efectos de aprendizaje sobre los participantes. Así, Jane Mansbridge (1999) escribió en un artículo pionero: “Tomar parte en las decisiones democráticas convierte a la mayoría de los participantes en mejores ciudadanos. Estoy convencida de ello porque mi experiencia lo confirma, pero no puedo probarlo y tampoco nadie lo ha hecho hasta ahora. [...] Los investigadores casi no han encontrado casos de estudio que les permitan medir las cualidades personales de los ciudadanos antes y después de la experiencia de participación, con el fin de evaluar si esta era la causa de determinados rasgos de su carácter. [...] Las investigaciones en ciencia política no alcanzan generalmente el grado de seriedad requerido en campos como la psicología, a causa de su débil validez interna, debida, a su vez, a la ausencia de un grupo de control o de un análisis de los efectos antes y después de un proceso. Una solución a estos problemas sería plantear un estudio que se apoyara en grupos de control aleatorios suficientemente grandes como para que incluso los pequeños efectos fueran significativos estadísticamente". Este llamamiento sería rápidamente escuchado. Los dispositivos sorteados que aparecen en esta época tienen la ventaja de constituir mini-laboratorios que permiten a los investigadores en ciencia política probar sus teorías de manera casi-experimental.

\section{La fascinación por el mini-público}

Aunque inicialmente era un teórico político, Fishkin $(1991 ; 1995)$ va a convertirse progresivamente en ingeniero jefe de la deliberación (Blondiaux, 2002). Antes que él, otros investigadores en ciencias sociales habían contribuido a poner en marcha experiencias cuasi-deliberativas, como Peter Dienel con las células de planificación en Alemania Occidental o Ned Crosby con los jurados ciudadanos, ambos en los años setenta. Aunque sus iniciativas tuvieron un importante éxito, contribuyendo a la difusión de los mini-públicos y del recurso al sorteo en política (Sintomer, 2012), no tenían una ambición teórica pro- 
piamente dicha, que aspirara a reubicar estos dispositivos en una nueva concepción de la democracia. James Fishkin ocupa, a la inversa, un lugar central en la comunidad científica de los deliberativistas. Una encuesta deliberativa, lo recuerdo, se basa en la constitución aleatoria de una muestra representativa de la población de alrededor de 400 personas. Los participantes, reunidos durante uno o dos fines de semana, alternan fases de formación, de discusión colectiva y de interrogación de un panel diversificado de expertos y representantes electos. Con la ayuda de cuestionarios administrados antes y después de la experiencia, Fishkin y sus colegas consiguieron demostrar que la deliberación era un vector de aprendizaje (Luskin, Fishkin, Jowell, 2002). La potencia del dispositivo -especialmente en comparación con la mayoría de los mini-públicos- se debe a la representatividad estadística de la muestra de ciudadanos, que permite a sus promotores generalizar los resultados al conjunto de la población (a la manera de los sondeos de opinión clásicos). La opinión de los participantes en la encuesta deliberativa es la que habría adoptado cualquier grupo de ciudadanos situado en condiciones similares.

La encuesta deliberativa es una experiencia completamente fabricada por un equipo de científicos -a menudo financiados por fundaciones y think tanks-; en principio no para ilustrar la toma de decisiones públicas, aunque los temas de discusión sean de interés público (reforma de la seguridad social, posición acerca de la seguridad nacional o sobre la entrada en la zona euro, etc.), sino para responder a una importante pregunta científica: ¿es la competencia política un dato heredado e inmutable o está influenciada por el contexto institucional en el que están situados los ciudadanos? Esta pregunta constituye uno de los principales temas de controversia en la ciencia política americana desde hace varios decenios (Blondiaux, 1996). Philip Converse (1964) había demostrado que, lejos de las pretensiones de la teoría clásica de la democracia, que hace del ciudadano un ser interesado en la cosa pública, la mayor parte de los individuos solamente tienen un conocimiento limitado de las cuestiones políticas y emiten opiniones cuanto menos fluctuantes (lo que Converse califica de "non-attitudes"4). En este sentido, los estudios empíricos de la deliberación ocupan inmediatamente un lugar central en la ciencia política americana, ya que responden, en parte, a algunas de estas cuestiones canónicas, relativas a la racionalidad de las elecciones políticas, especialmente electorales. Mientras que la demostración de la incompetencia política de las masas, que había dominado los trabajos durante treinta años, abría el camino a una interpretación elitista de la democracia, los estudios de la deliberación subrayan que es posible crear las condiciones institucionales para la formación de una ciudadanía ilustrada. En este sentido, las teorías deliberativas estaban inicialmente preñadas de una fuerza crítica, enfrentándose al paradigma elitista que postula la incompetencia de las masas. Intentar demostrar empíricamente que la competencia política es universal comporta innegablemente un aspecto subversivo en una ciencia política americana cuanto menos conservadora.

Los estudios de Fishkin, aunque rápidamente fueron criticados -especialmente en cuanto a la duración de los cambios de preferencias observados (Merkle, 1996)-, van a tener un importante desarrollo. A principios de los años dos mil, los estudios empíricos sobre deliberación a partir de dispositivos sorteados se multiplican (Gastil, Dillard,

4 "Falta de opiniones o actitudes" (N. del T.). 
1999; Hansen, Andersen, 2004; Grönlund et al., 2010). Los mini-públicos son rápidamente legitimados por la teoría deliberativa. Así, Archong Fung $(2003,339)$ afirma que "encarnan uno de los esfuerzos más constructivos para promover la democracia deliberativa". Jane Mansbridge (2010), pese a señalar ciertos límites, escribe por su parte: "La combinación de factores de representatividad y equilibrio, el vínculo con la decisión, la protección de los participantes y la inclusión hacen de la encuesta deliberativa el patrón oro de los procesos institucionales que tratan de crear deliberaciones informadas mediante una muestra representativa de ciudadanos". Algunos llegarán incluso a considerar a los mini-públicos como "el método más avanzado para institucionalizar la democracia deliberativa" (Estlub, 2014, 166). El auge de la deliberación en pequeños grupos sorteados ha sido tal, después de dos décadas, que podría tenerse la impresión de que los mini-públicos son la democracia deliberativa (para una perspectiva crítica véase Parkinson, 2006 y Curato, 2016).

Dos elementos aparecen como característicos de estas primeras investigaciones. Al principio, se concentran principalmente en el cambio de preferencias, recurriendo especialmente a estudios pre/post deliberación ${ }^{5}$. Se estudia así si la opinión de los participantes en la deliberación es diferente -mejor informada, más firme, más "tolerante" o "altruista"antes y después de la experiencia. En todos o casi todos los casos, se demuestra que la deliberación produce preferencias fundadas, confirmando que sirve para formar mejores ciudadanos (Gastil et al., 2010). Después, la deliberación se estudia principalmente sobre el terreno, en el marco de dispositivos impulsados no por los poderes públicos, sino por investigadores. Sin embargo, estos dispositivos cuasi-experimentales van a contribuir a legitimar el desarrollo paralelo de mini-públicos sorteados impulsados por actores institucionales o por la sociedad civil, con resultados dispares. Mientras tanto, el potencial crítico de la democracia deliberativa se verá muy atenuado.

\section{El auge de una industria del sorteo}

El éxito de esta empresa científica se materializa en la organización de "miles de deliberaciones moderadas y preparadas minuciosamente cada año en un país como los Estados Unidos" (Levine, 2014, 1), lo que es igualmente cierto en numerosos países de Europa y más allá. El éxito de este regreso del sorteo a la política se traduce -pero igualmente va acompañado, los dos fenómenos se alimentan- en la emergencia de un auténtico "mercado de la participación" (Mazeaud, Nonjon, 2015). Las administraciones locales en particular suelen llamar a consultores externos para diseñar y dirigir estos dispositivos. El objetivo de esta recuperación del sorteo en política consiste, especialmente, en superar la participación de los "sospechosos habituales", a saber, los ciudadanos politizados y a menudo comprometidos en estructuras asociativas o partidistas, para llegar a "ciudadanos ordinarios" y a las clases populares, menos inclinadas a participar. Aunque este objetivo puede interpretarse como voluntad de inclusión, va acompañado igualmente de intenciones

5 La evolución de las preferencias se estudia, en general, a partir de respuestas a cuestionarios, tratadas después estadísticamente. Para un método algo diferente, cualitativo, basado en el uso de la metodología Q, antes y después del mini-público, véase Niemeyer, 2011. 
más instrumentales, como la de evitar la intervención de grupos de interés y movimientos de oposición que podrían poner trabas a los planes de las instituciones. De manera más prosaica, el recurso al sorteo permite igualmente a las administraciones que quieren organizar procesos de participación disponer de un público cautivo, ofreciendo una solución al síndrome de la sala vacía (Gourgues, 2012). A pesar de que la elaboración de las muestras plantea a menudo dificultades ante el escaso interés de muchos ciudadanos cuando son seleccionados, el recurso al sorteo permite crear un público, dar voz al demos en acción; aunque esto pueda llegar a convertirse en un fin en sí mismo, sin que necesariamente se inscriba en un proyecto político preciso.

¿En qué medida este retorno al sorteo en política se ha traducido en una democratización de la democracia, como proyectaron sus promotores? Por un lado, la capacidad de organizar seria y eficazmente tales dispositivos ha contribuido a legitimar el uso del sorteo en otros espacios políticos. Así, durante las elecciones presidenciales francesas de 2017 varios candidatos de izquierda propusieron no solamente el recurso frecuente a los mini-públicos, sino igualmente, en algunos casos, la creación de una tercera cámara legislativa sorteada; una propuesta defendida desde mucho tiempo atrás por universitarios (Dahl, 1989; Sintomer, 2007), pero que hasta entonces apenas había tenido eco o que incluso había sido despreciada dentro de la esfera política. Esta recuperación del valor del sorteo ha significado también la aparición de colectivos ciudadanos, como \#mavoix, que experimentan con el sorteo de candidatos para las elecciones legislativas. El retorno al sorteo contribuye así a desnaturalizar el monopolio de la elección en la construcción de la representación.

Sin embargo, es necesario constatar que el recurso a los mini-públicos por parte de las instituciones después de veinte años está lejos de haber contribuido a profundizar la democracia. La mayor parte de ellos no tienen impacto sobre las decisiones públicas; tan solo emiten recomendaciones que quedan a menudo en letra muerta (Papadopoulos, Warin, 2007; Blondiaux, 2008). Aunque las instituciones puedan a veces adoptar una forma de "escucha selectiva" (Sintomer, Talpin, 2011; Font, Pasadas del Almo, Smith, 2016), eligiendo las propuestas que les parecen más pertinentes, al final siguen conservando el poder de decisión. No solamente no se ha modificado el reparto de poder, sino que tales experiencias contribuyen a reforzar la desconfianza de los ciudadanos respecto a la política, ya que se sienten a menudo engañados o instrumentalizados (Funes, Talpin, Rull, 2015). No obstante, hay experiencias excepcionales -como la Asamblea Ciudadana de la Columbia Británica en Canadá- que aspiran a unir el sorteo y la democracia directa. Así, la legalización mediante referéndum del matrimonio homosexual en Irlanda fue, en parte, el resultado de un proceso deliberativo sorteado que había formulado la propuesta previamente (Suiter, Farrell, Harris, 2016). Si bien tales excepciones atestiguan la potencial radicalidad del recurso al sorteo, la mayoría de las experiencias no ha logrado, hasta la fecha, modificar de manera duradera las decisiones públicas. Al contrario, el sorteo se revela a veces como una herramienta que los representantes electos usan para deslegitimar o adelantarse a la acción de asociaciones o movimientos sociales más radicales ${ }^{6}$. Como

6 Véase, por ejemplo, el estudio de Barbier, Bedu y Buclet (2009) sobre la instrumentalización de un jurado ciudadano en Bretaña, o Freschi y Mete (2009) en relación a un 21st century town meeting en Toscana. 
subrayan Caroline Lee y Zachary Romano: "lo que buscan en la deliberación los clientes y los entes que financian estos procesos es, sobre todo, una estrategia de gestión de los conflictos a los que se enfrentan cuando aparece una resistencia potencial o tangible a las medidas políticas de austeridad, procedente de la reorganización social, de los recortes de gastos del Estado y de la remodelación urbana" (Lee, Romano, 2013, 743). En definitiva, el sorteo, seleccionando a ciudadanos sin cualidades definidas, muy a menudo sin afiliación asociativa o partidaria, permitiría constituir un público dócil (Blondiaux, 2008). La manera en que se han servido de ello tanto la investigación sobre la democracia deliberativa como la mayor parte de los actores políticos ha contribuido a despolitizar el sorteo, haciendo de él un instrumento o un procedimiento exportable al precio de la pérdida de su radicalidad política.

A partir de un estudio centrado en el papel de los asesores en la animación de minipúblicos en Estados Unidos, Caroline Lee (2014) llega a importantes conclusiones. Señala especialmente los efectos de la ideología del small is beautiful, muy asentada en la industria participativa. Si bien el localismo de los dispositivos deliberativos permite a los ciudadanos "tocar con los dedos los efectos de su participación", al mismo tiempo son las élites políticas y económicas quienes toman, a otra escala, las grandes decisiones. La valorización de la acción local como medio para fomentar el cambio social se destruye así sistemáticamente. La suma de pequeñas victorias -cuando los dispositivos participativos las alcanzan- no conduce automáticamente a la justicia social y su articulación con otras escalas se revela como una etapa indispensable en el proceso. "El resultado de todos estos pequeños pasos hacia el empowerment no es, lamentablemente, un camino hacia la justicia social, sino una espiral asfixiante de resignación y retirada de la vida pública" (Lee, 2014, 226). Esta crítica al localismo de los mini-públicos coincide con algunos argumentos expuestos recientemente por teóricos de la deliberación, que denuncian un desplazamiento de la democracia deliberativa a la deliberación democrática encarnado por esta focalización en los dispositivos microscópicos.

\section{De la democracia deliberativa a la deliberación democrática}

Simone Chambers, en un artículo pionero (2009), subraya que los teóricos de la deliberación se han desplazado progresivamente desde un interés por la democracia deliberativa, es decir, centrado en la manera de hacer a la democracia más deliberativa, a la deliberación democrática, esto es, al estudio de las condiciones que permiten a una discusión, generalmente en pequeños grupos, respetar un cierto número de criterios procedimentales (inclusión, igual respeto, información, etc.). Desde su punto de vista, este interés por la deliberación en círculos restringidos se debe a la desconfianza de muchos deliberativistas hacia los discursos demagógicos y populistas. Así, una deliberación de calidad sería lisa y llanamente imposible en el espacio público ${ }^{7}$. Los deliberativistas habrían, por tanto, "abandonado la democracia de masas".

7 Esto es algo que discute Chambers (2009), quien defiende, al contrario, formas de retórica deliberativa a gran escala. 
Esta crítica ha animado a un cierto número de teóricos a precisar el tipo de articulación que plantean entre los dispositivos sorteados y la democracia de masas. Algunos defienden una función central para los mini-públicos, a semejanza de Archon Fung $(2007,159)$ : "Las reformas del espacio público ampliado pueden consistir principalmente en la multiplicación de mini-públicos, en lugar de intentar mejorar el gran público (o la opinión pública)". En la medida en que considera irreductible la oposición entre deliberación y participación de masas, James Fishkin (2009) ha llegado a veces a defender un papel decisorio para las encuestas deliberativas; una posición que es, sin embargo, muy minoritaria en este campo. En la medida en que las opiniones recogidas al final de la encuesta deliberativa son las que habría adquirido el gran público si hubiera podido participar y dado que la muestra de ciudadanos es estadísticamente representativa -un elemento esencial, en su opinión, ya que fundamenta la legitimidad democrática de su dispositivo, a diferencia de los jurados ciudadanos o las conferencias de consenso, por ejemplo-, sería legítimo que tales grupos pudieran tomar decisiones directamente. No obstante, Fishkin es consciente de que la centralidad de la noción de consentimiento en las concepciones ordinarias de la democracia hace que tal propuesta sea muy improbable.

Más allá de este argumento pragmático -los ciudadanos no querrían delegar su poder en un grupo de ciudadanos sorteados- esta propuesta ha sido igualmente juzgada como elitista. Lafont (2015) afirma así que si la legitimidad de los mini-públicos para decidir se debe a la calidad de su juicio o de las opiniones a las cuales llegan los ciudadanos después de la deliberación, eso supondría delegar el poder de decisión en expertos. Tal concepción epistémica de la legitimidad (Estlund, 2011) no puede, en efecto, apoyarse sobre el argumento de que los sorteados representan (estadística o sociológicamente) al conjunto de la población, ya que la experiencia deliberativa les ha transformado precisamente en seres diferentes del común de los mortales. En este sentido, los mini-públicos encarnarían una forma de "elitismo deliberativo" (Chambers, 2009; Pourtois, 2013).

Llevando la lógica fishkiniana hasta el final, Lafont (2015) se pregunta, desde una lógica contra-factual, si sería deseable, desde un punto de vista normativo, delegar el poder en una asamblea sorteada. Incluso aunque esta asamblea hubiera sido autorizada para gobernar -mediante un referéndum, por ejemplo-, tal escenario no sería democrático, ya que supondría la dominación de la masa de los ciudadanos por parte de seres informados. Estos inicialmente estarían próximos a los primeros, pero, al final, no tendrían mucho que ver con el pueblo y serían poco dados a rendir cuentas ante él. En tal sistema, los ciudadanos "seguirían ciegamente las opiniones y preferencias de sus delegados, en un mundo próximo pero inaccesible. Estarían dominados por su yo-ideal, pero dominados a pesar de todo". De acuerdo al principio de no-tiranía, Lafont considera ese escenario democráticamente insostenible.

¿Es necesario, por tanto, abandonar cualquier uso del sorteo dentro del sistema político? Muchos teóricos intentan, desde hace varios años, responder a esta cuestión e invitan a retomar la preocupación por la deliberación en el espacio público, en lugar de limitarse a pequeños espacios controlados. Una de las consecuencias de este giro "sistémico" es la marginación del sorteo en la teoría deliberativa. 


\section{La marginación del sorteo: el giro sistémico de la democracia deliberativa}

Frente a esta crítica a la centralidad que han tenido los mini-públicos en las investigaciones sobre deliberación, muchos teóricos defienden en la actualidad una concepción sistémica de la democracia deliberativa. El desafío ya no sería crear espacios deliberativos perfectos, pero aislados de la realidad social, sino introducir la deliberación en todos los niveles de la gobernanza democrática (Mansbridge, 1999; Parkinson y Mansbridge, 2012).

En este sentido, Lafont (2015) afirma que la simple multiplicación de dispositivos deliberativos sorteados no permitiría una democratización del espacio público. Efectivamente, incluso si estos posibilitan formular propuestas ilustradas y razonables, dado que el resto del público sigue estando poco informado, los mini-públicos no tendrán efecto. La Asamblea Ciudadana de la Columbia Británica se enfrentó a este tipo de dificultades. Aunque los ciudadanos sorteados se formaron gracias a su participación y llegaron a formular una propuesta de reforma original y razonable, no fueron capaces de influenciar a la opinión pública en general. Los ciudadanos que tuvieron conocimiento de la existencia de la Asamblea Ciudadana votaron mayoritariamente a favor en el referéndum, pero la mayoría del público la ignoraba; de modo que el referéndum no obtuvo el $60 \%$ de aprobación necesario para su ratificación (Lang, 2007; Warren y Pearse, 2008). Por tanto, parece difícil introducir la deliberación en el espacio público sin utilizar otros mecanismos más allá de los mini-públicos sorteados. Las investigaciones se concentran actualmente en la manera de reforzar la deliberación y la información en las campañas electorales y de referéndums (Gastil y Richards, 2013; Talpin, 2016b), dentro de los partidos políticos (Lefebvre y Roger, 2009) o en los medios de comunicación de masas (Parkinson, 2006; Girard, 2010). El sorteo se encuentra consecuentemente marginado en las reflexiones sobre la democracia deliberativa.

No obstante, esto no significa que no se le conceda ningún lugar en el sistema deliberativo. Sintomer (2012) afirma que los mini-públicos sorteados pueden cumplir cuatro tipos de funciones democráticas: aconsejar al príncipe mediante recomendaciones; evaluar la acción pública o una decisión; proponer proyectos de ley o de referéndum e informar a la opinión pública ${ }^{8}$. Por su parte, Niemeyer (2014) y Curato y Böker (2016) subrayan otras dos funciones que pueden caracterizar a los mini-públicos: la de brokers of knowledge, funcionando como intermediarios fiables entre los ciudadanos e informaciones complejas, y la de "escuelas de democracia", en las que los ciudadanos aprenden conocimientos y destrezas que podrán reinvertir en otros espacios. Böker y Elstub (2014) intentan por su parte radicalizar los mini-públicos para recuperar la filiación crítica de la teoría deliberativa. Promueven en particular la idea de dispositivos sorteados de abajo a arriba, autoorganizados por la sociedad civil y, por tanto, más autónomos de los poderes públicos, tanto en la definición de la agenda como en la gestión de las deliberaciones. Aunque tales experiencias puedan resultar interesantes, no resuelven el problema de su articulación con la toma de decisiones políticas y, en general, con el sistema deliberativo. Así lo muestran

8 Como es el caso de las Citizen Initiative Review en Oregón, cámaras sorteadas que facilitan información equilibrada a los ciudadanos antes de que se pronuncien sobre las propuestas sometidas a referéndum (Gastil y Richards, 2013). 
los dispositivos sorteados organizados por la sociedad civil en Islandia o en Bélgica estos últimos años (véase Reuchamps y Suiter, 2016). Curato y Böker $(2016,185)$ reconocen al final que el "enfoque sistémico requiere que los mini-públicos no jueguen un papel central, sino auxiliar, en la democratización deliberativa".

\section{6. ¿Recuperar la dimensión crítica de la teoría deliberativa mediante el sorteo?}

En un momento en que las lógicas plebiscitarias y autoritarias parecen imponerse en varias democracias liberales y la "posverdad" gana terreno, la defensa de la razón pública y de una concepción epistémica de la democracia por parte de los partidarios de la deliberación sigue siendo un horizonte político importante. A pesar de ello, ¿cómo no preguntarse por la creciente distancia entre las reflexiones académicas de los teóricos de la deliberación y el advenimiento de una era "posdemocrática" (Crouch, 2004), donde las principales decisiones se toman al margen de las instancias electas y lejos de la mirada del público? Se suponía que el giro sistémico de la teoría deliberativa debía tomar distancia con respecto al enfoque microscópico adoptado estos últimos años, con el fin de considerar las relaciones de poder en el análisis del funcionamiento democrático; pero uno no puede más que asombrarse ante la miopía de una parte de la literatura respecto a las dinámicas anti-deliberativas que estructuran hoy día el gobierno representativo. ¿Qué papel juega la deliberación frente a la influencia del dinero en las campañas electorales, la presión de las agencias de calificación financiera sobre los gobiernos o las prácticas de lobby en la toma de decisiones? (Bartels, 2009; Brown, 2015) En este momento, los teóricos de la deliberación prácticamente no se plantean estas preguntas. Por tanto, el sorteo, así como cualquier otra forma de inclusión política, parecen horizontes lejanos. Sin embargo, podemos preguntarnos, dado que el objetivo de la teoría deliberativa es introducir más discusión y reflexividad en el espacio público, si el sorteo no puede jugar otro papel menos accesorio y si no podría contribuir a radicalizar una teoría deliberativa que ha perdido parte de su potencial crítico.

Uno de los límites de las actuales teorías de la deliberación se debe a su concepción cooperativa de la decisión colectiva y del cambio social. Aunque ya existen reflexiones en este sentido (véase Mansbridge et al., 2010), podría considerarse un uso más agonístico del sorteo en el sistema deliberativo. Archon Fung y Eric Olin Wright (2003) defendieron hace más de quince años las virtudes de "contra-poderes deliberativos" capaces de interpelar a las instituciones para recordarles sus principios democráticos y deliberativos. No tanto, desde su punto de vista, para salvaguardar el equilibro del sistema, sino para asegurar el progreso social y la emancipación de las capas subalternas de la población. Sin embargo, esta intuición ha sido completamente obviada por la teoría deliberativa, quedando asociada a ciertos aspectos críticos de la democracia participativa, distinta de la deliberación (Pateman, 2012). Así, la principal obra del enfoque sistémico de la deliberación dedica solo dos páginas a los movimientos sociales (Parkinson y Mansbridge, 2012, 17-18), para subrayar su eventual contribución a las dimensiones epistémicas y democráticas del sistema. Resulta evidente que la acción colectiva sigue ocupando un lugar muy marginal en su conceptualización de la democracia. Esta ausencia es especialmente sorprendente en un momento en que las reivindicaciones democráticas y las 
prácticas deliberativas en asambleas son centrales en el movimiento altermundialista y en el "movimiento de las plazas" por todo el mundo (Della Porta, 2009; Ancelovici, Doufour y Nez, 2016).

El sorteo podría así convertirse, al mismo tiempo, en una herramienta para los movimientos sociales y en un elemento de radicalización del sistema deliberativo. Los contrapoderes deliberativos no tienen necesariamente que constituirse por sorteo. Su autonomía requiere la auto-organización y la auto-determinación de las formas que debe tomar la participación. Sin embargo, el sorteo puede jugar un papel importante. Para empezar, aunque esta vía apenas ha sido explorada hasta hoy, los movimientos sociales y los contrapoderes podrían recurrir al sorteo para ampliar su base social y reclutar de manera más o menos permanente nuevas caras. Eso les permitiría responder a una crítica que a menudo se les dirige para deslegitimarlos: su falta de representatividad. A continuación, una vez constituidos, tales espacios podrían contribuir a hacer surgir nuevas voces y perspectivas en el sistema deliberativo, cooperando en su dimensión epistémica. El sorteo de delegados dentro de esos espacios podría asimismo ayudar a combatir la "ley de hierro de la oligarquía" (Michels, 1910) y la dominación de una casta de representantes en su seno. Muchos movimientos sociales contemporáneos rechazan la representación y la designación de portavoces; el sorteo puede constituir un medio eficaz de expresión de sus aspiraciones horizontalistas y facilitar la rotación de líderes. En Francia, la experiencia de Nuit Debout fue debilitada por las veleidades vanguardistas de algunos de los actores más involucrados (Guichoux, 2016); el recurso al sorteo habría podido permitir conciliar el rechazo a los portavoces y la necesidad de organización. Los "movimientos de las plazas", en España y en Grecia, recurrieron a veces a tales mecanismos estos últimos años. En fin, pueden contemplarse otras formas de articulación entre contra-poderes y espacios instituidos de participación en los cuales se reúnan miembros sorteados. De hecho, es sorprendente que los teóricos de la democracia deliberativa se sientan actualmente tan poco inclinados a mecanismos como el sorteo de representantes, cuando la proliferación de las instancias de gobernanza multi-actores incita a ello (véase, sin embargo, Parkinson, 2012). Siguiendo algunas vías abiertas por otros (Fraser, 1992; Fung, 2011), me parece que una democracia realmente deliberativa no puede pasar sin contra-poderes estructurados que permitan luchar contra las tendencias plebiscitarias y autoritarias de las sociedades contemporáneas. En estos contra-poderes el sorteo puede reencontrar su radicalidad política.

\section{Referencias}

Ancelovici, M., Dufour, P. y Nez, H. (eds.) (2016): Street politics in the age of austerity: from the indignados to occupy, Amsterdam University Press, Ámsterdam.

Barber, B. (1984): Strong Democracy. Participatory Politics for a New Age, University of California Press, Berkeley.

Barbier, R., Bedu, C. y Buclet, N. (2009): "Portée et limites du dispositif jury citoyen", Politix, $\mathrm{n}^{\circ}$ 2, pp. 189-207.

Blondiaux, L. (1996): "Mort et résurrection de l'électeur rationnel. Les métamorphoses d'une problématique incertaine", Revue française de science politique, vol. 46, $\mathrm{n}^{\circ} 5$, pp. 753-791. 
Blondiaux, L. (2002): “Sondage et délibération”, Politix, n 57, pp. 167-180.

Blondiaux, L. (2008): Le nouvel esprit de la démocratie. Actualité de la démocratie participative, Seuil, París.

Böker, M. y Elstub, S. (2015): "The possibility of critical mini-publics: Realpolitik and normative cycles in democratic theory", Representation, vol. 51, $\mathrm{n}^{\circ} 1$, pp. 125-144.

Chambers, S. (2009): "Rhetoric and the public sphere: Has deliberative democracy abandoned mass democracy?", Political Theory, vol. 27, n 3, pp. 323-350.

Cohen, J. (1989): "Deliberation and Democratic Legitimacy", en A. Hamlin, P. Pettit P. (eds.): The Good Polity, Basil Blackwell, Oxford.

Converse, P. (1964): “The Nature of Belief Systems in Mass Publics”, en D. Apter (ed.): Ideology and Discontent, The Free Press, Nueva York.

Cossart, P., Felicetti, A. y Kloppenberg, J. (2016): "Introduction. Les town meetings, mythe fondateur de la démocratie américaine." Participations, $\mathrm{n}^{\circ}$ 2, pp. 5-47.

Crouch, C. (2004): Post-democracy, Polity, Cambridge.

Curato, N. y Böker, M. (2016): "Linking mini-publics to the deliberative system: a research agenda", Policy Sciences, vol. 49, n² 2, pp. 173-190.

Dahl, R. (1989): Democracy and its Critics, Yale University Press, New Haven.

Della Porta, D. (ed.) (2009): Democracy in Social Movements, Palgrave MacMillan, Basingstoke.

Elster, J. (1987): “The Market and the Forum”, en J. Bohman y W. Rehg W. (eds.): Deliberative Democracy. Essays on Reason and Politics, MIT Press, Cambridge.

Elster, J. (ed.) (1998): Deliberative Democracy, Cambridge University Press, Cambridge.

Elster, J. (1989): Solomonic judgements: Studies in the limitation of rationality, Cambridge University Press, Cambridge.

Estlund, D. (2011): L'autorité de la démocratie, Hermann, París.

Finley, M. I. (2003): Démocratie antique et démocratie moderne, Payot, París.

Fishkin, J. (1991): Democracy and Deliberation. New Directions for Democratic Reform, Yale University Press, New Haven.

Fishkin, J. (1995): The Voice of the People, Yale University Press, New Haven.

Fishkin, J. (2009): When the People Speak: Deliberative Democracy and Public Consultation, Oxford University Press, Oxford.

Font, J., Pasadas del Amo, S. y Smith, G. (2016): "Tracing the impact of proposals from participatory processes: methodological challenges and substantive lessons", Journal of Public Deliberation, $\mathrm{n}^{\circ} 12$, art. 1.

Fraser, N. (1992): "Rethinking the Public Sphere: A contribution to the Critique of Actually Existing Democracy”, en C. Calhoun (ed.): Habermas and the Public Sphere, The MIT Press, Cambridge.

Freschi, A. C. y Mete, V. (2009): “The political meanings of institutional deliberative Experiments. Findings on the Italian case", Sociologica, vol. 3, n 2-3.

Funes, M., Talpin, J. y Rull, M. (2014): "The Cultural Consequences of Engagement in Participatory Processes”, en J. Font, D. Della Porta y Yves Sintomer (eds.): Participatory Democracy in Southern Europe: Causes, Characteristics and Consequences, Rowman \& Littlefield, Londres, pp. 151-189. 
Fung, A. (2003): "Survey article: recipes for public spheres: eight institutional design choices and their consequences", Journal of political philosophy, vol. 1, n 3, pp. 338-367.

Fung, A. (2007): "Minipublics: Deliberative designs and their consequences", en S. Rosenberg (ed.): Deliberation, participation and democracy: Can the people govern, Palgrave McMillan, Londres, pp. 159-183.

Fung, A. (2005): "Deliberation before the Revolution", Political Theory.

Fung, A. y Wright, E. O. (2003): "Countervailing Power in Empowered Participatory Governance", en A. Fung, y E. O. Wright (eds.): Deepening Democracy, Institutional Innovations in Empowered Participatory Governance, Verso, Londres, pp. 259-289.

Gastil, J. y Dillard, J. (1999): "Increasing political sophistication through public deliberation", Political Communication, $\mathrm{n}^{\circ} 16$, pp. 3-23.

Gastil, J., Bacci, C. y Dollinger, M. (2010): "Is Deliberation Neutral? Patterns of Attitude Change During The Deliberative Polls", Journal of Public Deliberation, $\mathrm{n}^{\circ}$ 6, art. 3 .

Gastil, J. y Richards, R. (2013): "Making Direct Democracy Deliberative through Random Assemblies", Politics \& Society, vol. 41, n² 2, pp. 251-283.

Girard, C. (2010): L'idéal délibératif à l'épreuve des démocraties représentatives de masse: autonomie, bien commun et légitimité dans les théories contemporaines de la démocratie, Thèse pour le doctorat de philosophie, Université Paris 1.

Girard, C. (2011): "L'usage des références aux cités grecques dans les théories contemporaines de la délibération”, en J.-M. Werlings y F. Schulz (eds.): Débats antiques, Maison d'Archéologie et d'Ethnologie René-Ginouvès, París, pp. 141-153.

Goodwin, B. (2011): "Utiliser le hasard contre la confiscation du pouvoir", Esprit, n 8 , pp. $145-152$.

Gourgues, G. (2012): “Des dispositifs participatifs aux politiques de la participation. L'exemple des conseils régionaux français", Participations, $n^{\circ} 2$, pp. 30-52.

Grönlund, K., Setälä, M. y Herne, K. (2010): "Deliberation and civic virtue: lessons from a citizen deliberation experiment", European Political Science Review, vol. 2, n 1, pp. 95-117.

Guichoux, A. (2016): "Nuit debout et les mouvements des places désenchantement et ensauvagement de la démocratie", Les Temps Modernes, n 5, pp. 30-60.

Habermas, J. (1981): Théorie de l'agir communicationnel, 2 tomos, Fayard, París.

Hansen, K. y Andersen, V. (2004): "Deliberative democracy and the deliberative poll on Euro", Scandinavian political studies, vol. 27, n 3, pp. 261-286.

Lafont, C. (2015): "Deliberation, Participation, and Democratic Legitimacy: Should Deliberative Mini-publics Shape Public Policy?", Journal of Political Philosophy, vol. 23, $\mathrm{n}^{\circ} 1$, pp. 40-63.

Lang, A. (2007): “But Is It for Real? The British Columbia Citizens' Assembly as a Model of State-Sponsored Citizen Empowerment”, Politics \& Society, vol. 35, n 1, pp. 35-69.

Lee, C. (2014): Do it Yourself Democracy. The Rise of the Public Engagement Industry, Oxford University Press, Oxford.

Lee, C. y Romano, Z. (2013): "Democracy's new discipline: Public deliberation as organizational strategy", Organization Studies, vol. 34, n 5-6, pp. 733-753.

Lefebvre, R. y Roger, A. (2009): Les partis politiques à l'épreuve des procédures délibératives, Presses Universitaires de Rennes, Rennes. 
Luskin, R. C., Fishkin, J. S. y Jowell, R. (2002): “Considered Opinions: Deliberative Polling in Britain", British Journal of Political Science, n 32, pp. 455-487.

Manin, Bernard (1985): "Volonté générale ou delibération ? Esquisse d'une théorie générale de la délibération politique", Le débat, $\mathrm{n}^{\circ} 33$.

Mansbridge, J. (1999a): "On the idea that participation makes better citizens", en S. Elkin y K. Soltan (eds.): Citizen Competence and Democratic Institutions, The Pennsylvania University press, Filadelfia.

Mansbridge, J. (1999b): "Everyday Talk in the Deliberative System", en S. Macedo (ed.): Deliberative Politics: Essays on Democracy and Disagreement, Oxford University Press, Oxford.

Mansbridge, J. (2010): "Deliberative polling as the gold standard", The Good Society, vol. $19, \mathrm{n}^{\circ} 1$, pp. 55-62.

Mansbridge, J., Bohman, J., Chambers, S., Estlund, D., Føllesdal, A., Fung y Marti, J. L. (2011): "La place de l'intérêt particulier et le rôle du pouvoir dans la démocratie délibérative", Raisons politiques, $\mathrm{n}^{\circ} 2$, pp. 47-82.

Mazeaud, A. y Nonjon, M. (2015): "De la cause au marché de la démocratie participative", Agone, $\mathrm{n}^{\circ}$ 1, pp. 135-152.

Merkle, D. M. (1996): “The Polls - Review. The National Issues Convention Deliberative Poll", Public Opinion Quarterly, n 60, pp. 588-619.

Michels, R. (1910): Les partis politiques. Essai sur les tendances oligarchiques de la démocratie, Payot, París.

Niemeyer, S. (2011): “The emancipatory effect of deliberation: Empirical lessons from minipublics", Politics \& Society, vol. 39, n 1, pp. 103-140.

Papadopoulos, Y. y Warrin, P. (2007): “Are innovative, participatory and deliberative procedures in policy making democratic and effective?", European journal of political research, vol. 46, $\mathrm{n}^{\circ} 4$, pp. 445-472.

Parkinson, J. y Mansbridge, J. (eds.): Deliberative Systems: Deliberative Democracy at the Large Scale, Cambridge University Press, Cambridge.

Parkinson, J. (2006): "Rickety bridges: using the media in deliberative democracy", British Journal of Political Science, vol. 36, n 1, pp. 175-183.

Parkinson, J. (2012): "Democratizing Deliberative Systems", en J. Parkinson y J. Mansbridge (eds.): Deliberative Systems: Deliberative Democracy at the Large Scale, Cambridge University Press, Cambridge, pp. 153-171.

Pateman, C. (2012): "Participatory Democracy Revisited", Perspectives on politics, vol. 10, $\mathrm{n}^{\circ} 1, \mathrm{pp} .7-19$.

Pourtois, H. (2013): "Mini-publics et démocratie délibérative", Politique et Sociétés, vol. $32, \mathrm{n}^{\circ} 1$, pp. 21-41.

Rancière, J. (2005): La haine de la démocratie, La Fabrique, París.

Reuchamps, M. y Suiter, J. (eds.) (2016): Constitutional Deliberative Democracy in Europe, ECPR Press, Colchester.

Sintomer, Y. (2007): Le pouvoir au peuple. Tirage au sort, jurys citoyens et démocratie participative, La Découverte, París.

Sintomer, Y. (2011): “Tirage au sort et politique: de l'autogouvernement républicain à la démocratie délibérative", Raisons politiques, n² 2, pp. 159-186. 
Sintomer, Y. y Talpin, J. (eds.) (2011): La démocratie participative au-delà de la proximité: le Poitou-Charentes et l'échelle régionale, Presses Universitaires de Rennes, Rennes.

Sintomer, Y. (2012): Petite histoire de l'expérimentation démocratique. Tirage au sort et politique d'Athènes à nos jours, La Découverte, París.

Stone, P. (2009): “The logic of random selection", Political theory, vol. 37, n 3, pp. 375397.

Suiter, J., Farrell, D. y Harris, C. (2016): “The Irish constitutional convention. A case of high legitimacy ?”, en M. Reuchamps y J. Suiter (eds.): Constitutional Deliberative Democracy in Europe, ECPR Press, Colchester.

Talpin, J. (2016): "How can constitutional reforms be deliberative ? The hybrid legitimaticies of constitutional deliberative democracy", en M. Reuchamps y J. Suiter (eds.): Constitutional Deliberative Democracy in Europe, ECPR Press, Colchester.

Warren, M. y Pearse, H. (eds.) (2008): Designing Deliberative Democracy. The British Columbia Citizen Assembly, Cambridge University Press, Cambridge. 
